\section{H I G H L I G H T S}

\section{IN BRIEF}

\section{FUNCTIONAL GENOMICS}

Inferring genetic networks and identifying compound mode of action via expression profiling.

Gardner, T. S. et al. Science 301, 102-105 (2003)

Gardner et al. present a new model — network identification by multiple regression (NIR) - that provides a framework for elucidating the structure and function of genetic networks and identifying the molecular targets of uncharacterized pharmacological compounds. Using systematic transcriptional perturbations, the authors show that NIR correctly identified the main regulatory connections and transcriptional targets of mitomycin C activity in a nine-gene subnetwork of the Escherichia coli SOS pathway.

\section{DEVELOPMENTAL GENETICS}

Suppression of ovarian follicle activation in mice by the transcription factor Foxo3a.

Castrillon, D. H. et al. Science 301, 215-218 (2003)

The authors found that female Foxo $3 a^{-/-}$mice developed premature ovarian failure (POF) and were infertile by the time they were 15 -weeks old. As the forkhead transcription factor Foxo3a is crucial for the suppression of ovarian follicle activation, and accelerated follicle activation can lead to POF in mice, they suggest that a similar mechanism could contribute to the depletion of follicles that is seen in women with POF.

\section{GENE REGULATION}

Anchor cell invasion into the vulval epithelium in C. elegans.

Sherwood, D. R. \& Sterberg, P. W. Dev. Cell 5, 21-31 (2003)

Cell-invasive behaviour is vital during development, but is also associated with pathologies, such as cancer. This paper introduces the first genetic model system to allow this process to be visualized and manipulated in vivo, based on the Caenorhabditis elegans anchor cell (AC), which initiates the formation of the uterine-vulval connection through a cellinvasion event. The study implicates several gene networks in the control of AC invasion, and provides assays with which to identify the genes involved.

\section{EVOLUTION}

Widespread horizontal transfer of mitochondrial genes in flowering plants.

Bergthorsson, U. et al. Nature 424, 197-201 (2003)

Horizontal gene transfer (HGT) was thought to be rare among higher eukaryotes. However, the authors present strong evidence for the frequent exchange of mitochondrial genes encoding ribosomal and respiratory proteins between distantly related species during angiosperm evolution. This has important consequences for plant molecular phylogenetics and raises questions about the risk of transgene escape from genetically modified plants.

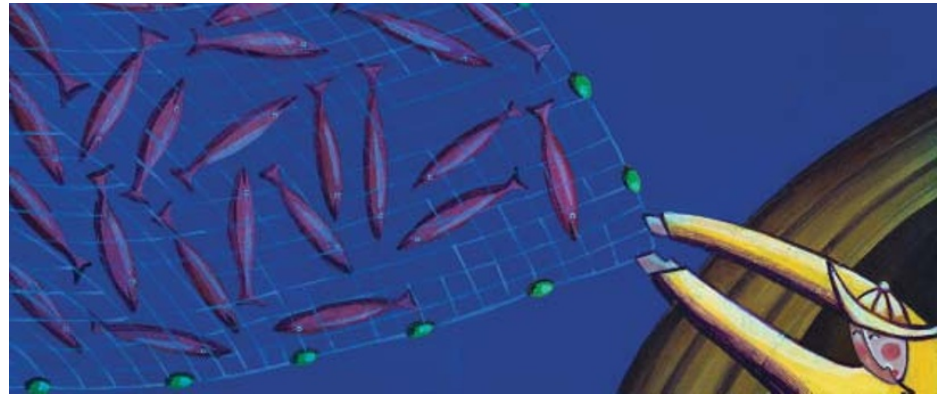

EVOLUTION

\title{
Closing the net on chordate origins
}

Despite much investigation, the origin of the phylum Chordata, which contains all vertebrates including ourselves, remains unclear. The central nervous system (CNS) could hold the answers. So, solving the mystery of its emergence will shed light on the evolutionary history and phylogenetic placement of the chordates.

However, the chordate CNS, in which neurons are concentrated in the brain and nerve cord, has variously been proposed to originate from an ancestor with a dorsal-centralized, ventral-centralized or non-centralized nerve network. These theories, which are all consistent with recent molecular phylogenies, are clearly mutually incompatible.

Lowe et al. have tackled this problem with a comparative study of gene expression in chordates and the hemichordate acorn worm Saccoglossus kowalevskii. The hemichordates, along with the echinoderms, are the closest relatives of the chordates, and together these three phyla constitute the deuterostomes. The diffuse nerve net of Saccoglossus, which lacks a brain, could therefore represent either the ancestral deuterostome condition or the secondary loss of an ancestral CNS.

To investigate these options, the authors isolated Saccoglossus orthologues of 22 genes involved in the anterioposterior patterning of the chordate CNS, the domains of expression of which have been mapped in the chordate neural plate and nervous system. All were strongly expressed in the ectoderm of Saccoglossus.

Detailed mapping of expression patterns showed that despite differences in the overlap of genes involved in boundary formation in the chordate brain, such as orthodenticle-like $(O T X)$ and engrailed $(E N)$, the overall organization was highly similar: orthologues of genes expressed in the chordate forebrain had anterior expression domains in Saccoglossus, whereas those expressed in the chordate midbrain, hindbrain and spinal cord were expressed in progressively more posterior regions of its body.

So, although the nervous system of Saccoglossus lacks morphological complexity, it is highly organized with respect to its gene-expression patterns, which parallel those of advanced chordates. This indicates conserved domain topography among the deuterostomes, which could pre-date the emergence of the CNS.

Moreover, Lowe et al. found that 14 of the 22 conserved expression domains in deuterostomes had similar locations in one or more protostomes (the group that contains all other higher animal phyla). They argue that these similarities are most parsimoniously explained as conservation of the expression patterns of a shared common ancestor of both groups.

This is the first molecular evidence to show that the ancestor of the deuterostomes, and perhaps even the protostomes, might have had a diffuse nerve net with a highly structured underlying gene-expression map from which the complex CNS of species such as humans and Drosophila could have independently evolved - a theory that has been largely overlooked.

These data are not unequivocal, and the analysis of relevant outgroups should provide important confirmation. Nonetheless, Lowe et al. have revealed an unexpected evolutionary flexibility in the nervous system, and their gene-expression maps offer an exciting new perspective on the evolution of the CNS that will help to pinpoint the elusive origin of the chordates.

Victoria Kitchener

(2) References and links ORIGINAL RESEARCH PAPER Lowe, C. J. et al. Anteroposterior patterning in hemichordates and the origins of the chordate nervous system. Cell 113, 853-865 (2003) 